\title{
Pearls
}

\section{Pearls: Clinical Application of Ranawat's Sign}

\author{
Thomas J. Blumenfeld MD
}

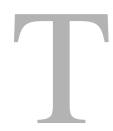
he risk of dislocation after THA has decreased in part due to enhanced repair of the capsule, larger femoral heads, and refining our surgical techniques [1]. Still, dislocation remains one of the more difficult complications for the patient to manage and for the surgeon to treat. A stable, impingement-free total hip requires the surgeon to position the components correctly,

A note from the Editor-in-Chief: We are pleased to present the next installment of "Pearls", a column in Clinical Orthopaedics and Related Research ${ }^{\mathbb{R}}$. In this column, distinguished surgeons, scientists, or scholars share surgical or professional tips they use to help surmount important or interesting problems. We welcome reader feedback on all of our columns and articles; please send your commentstoeic@clinorthop.org.

The author certifies that neither he, nor any members of his immediate family, have any commercial associations (such as consultancies, stock ownership, equity interest, patent/licensing arrangements, etc) that might pose a conflict of interest in connection with the submitted article. All ICMJE Conflict of Interest Forms for authors and Clinical Orthopaedics and Related Research ${ }^{\mathbb{R}}$ editors and board members are on file with the publication and can be viewed on request.

The opinions expressed are those of the writers, and do not reflect the opinion or policy of $C O R R^{\circledR}$ or The Association of Bone and Joint Surgeons ${ }^{\circledR}$.

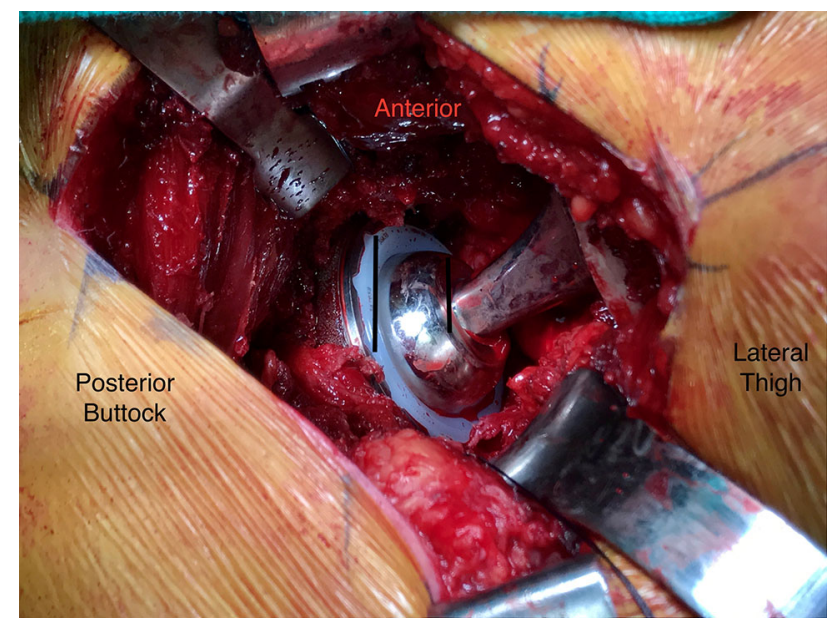

Fig. 1 This figure shows the right hip. The smaller black line is placed across the base of the femoral head, and the larger black line is across the face of the neutral liner. The operated leg has been internally rotated $45^{\circ}$ while in extension. Because both lines are parallel, a combined $45^{\circ}$ of anteversion can be identified. (Published with permission from Thomas J. Blumenfeld MD).

particularly with respect to anteversion; this anteversion can be considered a product of both femoral and acetabular anteversion.

In 1994, Drs. Lucas and Scott [2] introduced the Ranawat sign as an intraoperative maneuver to assess component positioning. In this

\section{T. J. Blumenfeld MD ( $\square)$}

Sutter Medical Center, 2801 K Street,

Suite 500, Sacramento, CA 95816, USA

e-mail: tblumenfeld59@gmail.com approach, the operated leg is placed in extension and internally rotated $45^{\circ}$. If the base of the femoral head is parallel to the acetabulum, a combined $45^{\circ}$ of anteversion should be identified (Fig. 1). The Ranawat sign can help identify whether the acetabulum is retroverted or excessively anteverted relative to the femoral prosthesis.

In order to achieve success with the Ranawat sign, surgeons must preoperatively stabilize the pelvis. With the patient in the lateral decubitus 


\section{Pearls}

position, I carefully position the pelvis in a nine-piece hip-holder set, and feel both anterior and superior iliac spines. I prefer the operative side (upper spine) to be $10^{\circ}$ posterior to the inferior spine because when the femur is retracted anteriorly during the surgery, some anterior pelvic tilt occurs. Next, I feel both iliac crests and determine if they are parallel (no inclination change), tilting towards the operated side (thus requiring me to drop my hand further down when impacting the cup to avoid excessive inclination), or tilting away (requiring me to raise my hand during impaction to avoid a horizontal placement of the acetabular component).

In addition to providing information on combined anteversion, Ranawat's sign also can serve a second purpose, which was not described in the original publication: Identifying acetabular inclination. The operated leg is extended, internally rotated 45 degrees, and kept parallel to the floor. If the iliac crests are directly atop one another and perpendicular to the floor, with a prosthetic neck shaft angle of $135^{\circ}$, the finding of the prosthetic neck being perpendicular to the face of the acetabulum suggests an acetabular inclination of approximately $45^{\circ}$ (Fig. 2).

There are three pitfalls in the use of this clinical sign. First, if the pelvic position changes during surgery, then what is visually identified may be

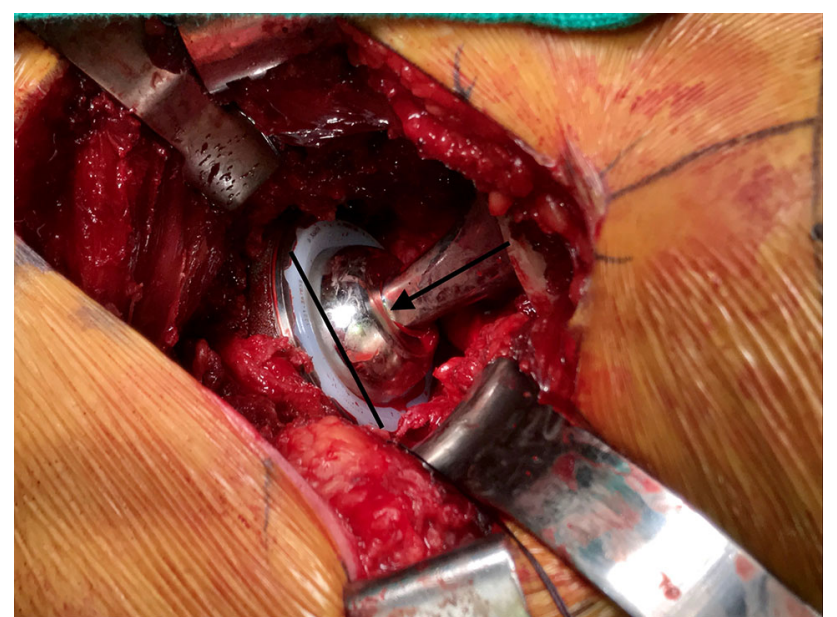

Fig. 2 This figure shows the right hip. The black line is placed along the superior inferior, or inclination axis of the acetabulum. The black arrow sits along the prosthetic neck of the femoral component, which has a $135^{\circ}$ neck-shaft angle. The operated leg is in extension, and parallel to the floor. Because these lines are perpendicular, the acetabular inclination is approximately $45^{\circ}$. (Published with permission from Thomas J. Blumenfeld MD).

misleading. For example, if the pelvis falls anteriorly during surgery, the acetabulum will appear excessively anteverted. Second, Ranawat's sign does not provide the exact anteversion of the components, (nor whether one is excessively anteverted); it only provides the sum of the two components. Third, if the prosthetic hip subluxates or dislocates during intraoperative ROM testing, the surgeon must identify a cause and correct it, regardless of what Ranawat's sign may or may not suggest in terms of component alignment.

While advances in technology and surgical technique are important, most surgeons rely on experience and feel when evaluating the stability of a total hip. Ranawat's sign is an easy addition to the intraoperative tests surgeons can use to achieve the goal of a stable THA.

\section{References}

1. Akshay G, Lau EC, Ong KL, Berry DJ, Malkani AL. Dislocation rates following primary total hip arthroplasty have plateaued in the Medicare population. J Arthroplasty. 2015;30: 743-746.

2. Lucas DH, Scott RD. The Ranawat sign-a specific maneuver to assess component positioning in total hip arthroplasty. J Orthop Techniques. 1994;2:59-61. 